\title{
Consecutive Rosochatius Deformations of the Garnier System and the Hénon-Heiles System
}

\author{
Baoqiang Xia and Ruguang Zhou \\ School of Mathematics and Statistics, Jiangsu Normal University, Xuzhou, Jiangsu 221116, China \\ Correspondence should be addressed to Baoqiang Xia; xiabaoqiang@126.com
}

Received 8 January 2014; Accepted 20 February 2014; Published 31 March 2014

Academic Editor: Weiguo Rui

Copyright ( 2014 B. Xia and R. Zhou. This is an open access article distributed under the Creative Commons Attribution License, which permits unrestricted use, distribution, and reproduction in any medium, provided the original work is properly cited.

An algorithm of constructing infinitely many symplectic realizations of generalized sl(2) Gaudin magnet is proposed. Based on this algorithm, the consecutive Rosochatius deformations of integrable Hamiltonian systems are presented. As examples, the consecutive Rosochatius deformations of the Garnier system and the Hénon-Heiles system as well as their Lax representations, are obtained.

\section{Introduction}

Usually the integrability of a Hamiltonian system is destroyed even with a very small perturbation. As early as in 1877 , Rosochatius first discovered that it would keep the integrability to add a potential of the sum of inverse squares of the coordinates to that of the Neumann system $[1,2]$. This provides an interesting example of integrable perturbation. Nowadays, the resulting system is called the NeumannRosochatius system [3-7]. In 1985, Wojciechowski gained an analogy system (called Garnier-Rosochatius system) for the Garnier system $[8,9]$. Later in 1999, based on the Deift technique and a well-known theorem that the Gauss map transforms the Neumann system to the Jacobi system, Kubo et al. constructed the analogy system for the Jacobi system or the geodesic flow equation on the ellipsoid [10-12]. In 2007, one of the authors (Zhou) generalized the Rosochatius deformations of the constrained soliton flows [13], and then the method has been extended to construct the integrable deformations of the symplectic maps [14] and the soliton equations with self-consistent sources [15].

There appear some important physical and mathematical applications of Rosochatius deformed integrable systems. For example, the Neumann-Rosochatius system can be used to describe the dynamics of a rotating closed string and the membranes [16, 17], the Garnier-Rosochatius system can be used to solve the multicomponent coupled nonlinear Schödinger equation [18, 19], and the Rosochatius deformation of the $\mathrm{KdV}$ equation with self-consistent sources can be used to establish the bi-Hamiltonian structure of the KdV6 equation [20].

Recently, we proposed an approach to generate integrable Rosochatius deformations of the Neumann system consecutively [21]. The Lax matrix of the $N$-copies of Neumann system is of the form of classical sl(2) Gaudin magnet defined on the 2(N-1)-dimensional submanifold. In this paper, we would like to show that the approach can be applied to the integrable Hamiltonian systems whose Lax matrices are of the form of the generalized Gaudin magnet. We first present an algorithm of constructing infinitely many realizations of generalized sl(2) Gaudin magnet model. Then, we describe how to generate integrable Hamiltonian systems based on the realizations of sl(2) Gaudin magnet. The Rosochatius deformation of an integrable Hamiltonian system is explained as a special case of the realizations of generalized $\mathrm{sl}(2)$ Gaudin magnet model. Thus, such an algorithm enables us to construct Rosochatius deformations of the integrable Hamiltonian systems consecutively. As applications, we obtain the consecutive Rosochatius deformations of the Garnier system and the Hénon-Heiles system as well as their Lax representations.

The plan of the paper is as follows. In Section 2, we propose infinitely many symplectic realizations of sl(2) Gaudin magnet and describe how to generate the integrable Hamiltonian systems based on these realizations. In Sections 3 and 4 , we pay attention to studying the integrable deformations 
of the Garnier system and Hénon-Heiles system, respectively. Some concluding remarks are drawn in Section 5.

\section{The Generalized sl(2) Gaudin Magnet and Its Realizations}

2.1. The Realizations of the Generalized sl(2) Gaudin Magnet. We consider the Lax matrix of the form of the generalized Gaudin magnet $[22,23]$

$$
L(\lambda)=L_{0}(\lambda)+\frac{1}{2} \sum_{j=1}^{N} \frac{1}{\lambda-\lambda_{j}}\left(\begin{array}{cc}
S_{j}^{0} & S_{j}^{-} \\
S_{j}^{+} & -S_{j}^{0}
\end{array}\right),
$$

where $L_{0}(\lambda)$ is a traceless $2 \times 2$ matrix whose entries are polynomials of $\lambda$ or $\lambda^{-1}$ and $S_{j}$ satisfy $N$ copies of the standard sl(2) algebra

$$
\begin{array}{r}
\left\{S_{j}^{0}, S_{k}^{+}\right\}=2 \delta_{j k} S_{k}^{+}, \quad\left\{S_{j}^{0}, S_{k}^{-}\right\}=-2 \delta_{j k} S_{k}^{-}, \quad\left\{S_{j}^{+}, S_{k}^{-}\right\}=4 \delta_{j k} S_{k}^{0}, \\
j, k=1,2, \ldots, N,
\end{array}
$$

with $N$ Casimirs

$$
C_{j}=\left(S_{j}^{0}\right)^{2}+S_{j}^{-} S_{j}^{+}, \quad j=1,2, \ldots, N .
$$

It is well known that the sl(2) algebra (2) has a symplectic realization:

$$
\begin{array}{r}
S_{j}^{0}=q_{j} p_{j}, \quad S_{j}^{-}=-q_{j}^{2}, \quad S_{j}^{+}=p_{j}{ }^{2}, \\
j=1,2, \ldots, N,
\end{array}
$$

where $q_{j}, p_{j}$ are canonical coordinates on the standard symplectic space $\left(\mathbb{R}^{2 N}, \omega^{2}=\sum_{j=1}^{N} d p_{j} \wedge d q_{j}\right)$. Under the realization of (4), the Lie-Poisson brackets (2) are recovered by computing the standard Poisson bracket

$$
\{F(q, p), G(q, p)\}=\sum_{j=1}^{N}\left(\frac{\partial F}{\partial q_{j}} \frac{\partial G}{\partial p_{j}}-\frac{\partial F}{\partial p_{j}} \frac{\partial G}{\partial q_{j}}\right),
$$

where $q=\left(q_{1}, \ldots, q_{N}\right), p=\left(p_{1}, \ldots, p_{N}\right), F(q, p)$, and $G(q, p)$ are arbitrary smooth functions about $q$ and $p$.

With a direct calculation, we observe the following proposition.

Proposition 1. If $S_{j}^{0}=f_{j}(q, p), S_{j}^{-}=g_{j}(q, p)$, and $S_{j}^{+}=$ $h_{j}(q, p)$ is a realization of $(2)$, so are

$$
\begin{gathered}
\widetilde{S}_{j}^{0}=f_{j}(q, p), \quad \widetilde{S}_{j}^{-}=g_{j}(q, p), \\
\widetilde{S}_{j}^{+}=h_{j}(q, p)+\gamma_{j} g_{j}^{-1}(q, p), \\
\widehat{S}_{j}^{0}=f_{j}(q, p), \quad \widehat{S}_{j}^{-}=g_{j}(q, p)+\beta_{j} h_{j}^{-1}(q, p), \\
\widehat{S}_{j}^{+}=h_{j}(q, p),
\end{gathered}
$$

where $\gamma_{j}, \beta_{j},(j=1,2, \ldots, N)$ are arbitrary constants.
This proposition provides us with two kinds of new realizations of sl(2) algebra (2) from a known one. Moreover, applying such two kinds of realizations in turn, we can construct an infinitely many realizations of sl(2) algebra (2). For example, from (4), we obtain the following realizations of (2):

$$
\begin{array}{ll}
\widetilde{S}_{j}^{0}=q_{j} p_{j}, & \widetilde{S}_{j}^{-}=-q_{j}^{2}, \quad \widetilde{S}_{j}^{+}=p_{j}^{2}+\gamma_{j} q_{j}^{-2}, \\
\widehat{S}_{j}^{0}=q_{j} p_{j}, & \widehat{S}_{j}^{-}=-q_{j}^{2}-\beta_{j}\left(p_{j}^{2}+\gamma_{j} q_{j}^{-2}\right)^{-1}, \\
& \widehat{S}_{j}^{+}=p_{j}^{2}+\gamma_{j} q_{j}^{-2} .
\end{array}
$$

2.2. A Recipe for Generating Integrable Hamiltonian Systems Based on Realizations of sl(2) Gaudin Magnet. Now, we describe how to generate an integrable Hamiltonian system based on a symplectic realization of sl(2) Gaudin magnet. We suppose that the Lax matrix (1) satisfies an $r$-matrix relation [24]

$$
\left\{L_{1}(\lambda), L_{2}(\mu)\right\}=\left[r_{12}(\lambda, \mu), L_{1}(\lambda)\right]-\left[r_{21}(\lambda, \mu), L_{2}(\mu)\right],
$$

where $L_{1}(\lambda)=L(\lambda) \otimes I_{2}, L_{2}(\mu)=I_{2} \otimes L(\mu), I_{2}$ is $2 \times 2$ identity matrix, $\mu$ is an arbitrary parameter, and $[\cdot, \cdot]$ denotes the commutator of the matrices, such as $\left[r_{12}, L_{1}\right]=r_{12} L_{1}-L_{1} r_{12}$. According to the general theory of the $r$-matrix $[24,25]$, we have

$$
\{\operatorname{det} L(\lambda), \operatorname{det} L(\mu)\}=0 \text {. }
$$

First, we expand $\operatorname{det} L(\lambda)$ as

$$
\operatorname{det} L(\lambda)=\sum_{j=k_{0}}^{\infty} F_{j} \lambda^{-j}, \quad \text { or } \quad \operatorname{det} L(\lambda)=\sum_{j=k_{0}}^{\infty} F_{j} \lambda^{j} .
$$

From (10), we have

$$
\left\{F_{j}, F_{k}\right\}=0, \quad j, k \geq k_{0},
$$

which implies that $F_{k}$ 's are in involution in pairs. Usually, we can single out $N$ functionally independent $F_{k_{1}}, \ldots, F_{k_{N}}$ among $\left\{F_{k}\right\}_{k \geq k_{0}}$. Choosing a Hamiltonian $H$, which is composed of some of $F_{k}$ 's, we have

$$
\left\{H, F_{k_{j}}\right\}=0, \quad 1 \leq j \leq N .
$$

Functionally independent and involutive pairwise integrals, $F_{k_{1}}, \ldots, F_{k_{N}}$, ensure that the Hamiltonian system $H$ is completely integrable in the sense of Liouville [26].

Further, substituting a realization of (2) into the Lax matrix (1) and the corresponding $F_{k}$ 's and $H$ defined above, we finally obtain an integrable Hamiltonian system with the Hamiltonian $H$ expressed in canonical coordinates $\left(q_{j}, p_{j}\right)$ :

$$
q_{j, x}=\frac{\partial H}{\partial p_{j}}, \quad p_{j, x}=-\frac{\partial H}{\partial q_{j}}, \quad 1 \leq j \leq N .
$$

The above recipe shows that, once having a symplectic realization of $\mathrm{sl}(2)$ algebra (2), we may obtain an integrable Hamiltonian system. In the next sections, we will 
take the Garnier system and the Hénon-Heiles system as examples to show that the Rosochatius deformations and second Rosochatius deformations of integrable systems can be generated from the realizations of (7) and (8), respectively, according to the above recipe. Thus, applying Proposition 1 in turn enables us to consecutively construct Rosochatius deformations of the integrable Hamiltonian systems.

\section{Consecutive Rosochatius Deformations of the Garnier System}

We take $L_{0}(\lambda)$ in (1) as

$$
L_{0}(\lambda)=\left(\begin{array}{cc}
0 & 1 \\
-\lambda+\frac{1}{2} \sum_{j=1}^{N} S_{j}^{-} & 0
\end{array}\right) ;
$$

then, the Lax matrix (1) becomes

$$
\begin{aligned}
L(\lambda) & =\left(\begin{array}{cc}
0 & 1 \\
-\lambda+\frac{1}{2} \sum_{j=1}^{N} S_{j}^{-} & 0
\end{array}\right)+\frac{1}{2} \sum_{j=1}^{N} \frac{1}{\lambda-\lambda_{j}}\left(\begin{array}{cc}
S_{j}^{0} & S_{j}^{-} \\
S_{j}^{+} & -S_{j}^{0}
\end{array}\right) \\
& \triangleq\left(\begin{array}{cc}
L^{11}(\lambda) & L^{12}(\lambda) \\
L^{21}(\lambda) & -L^{11}(\lambda)
\end{array}\right) .
\end{aligned}
$$

Direct calculations yield that

$$
\begin{aligned}
& \left\{L^{11}(\lambda), L^{11}(\mu)\right\}=\left\{L^{12}(\lambda), L^{12}(\mu)\right\}=0, \\
& \left\{L^{21}(\lambda), L^{21}(\mu)\right\}=2 L^{11}(\lambda)-2 L^{11}(\mu), \\
& \left\{L^{11}(\lambda), L^{12}(\mu)\right\}=\frac{1}{\mu-\lambda}\left(L^{12}(\mu)-L^{12}(\lambda)\right), \\
& \left\{L^{12}(\lambda), L^{21}(\mu)\right\}=\frac{2}{\mu-\lambda}\left(L^{11}(\mu)-L^{11}(\lambda)\right), \\
& \left\{L^{11}(\lambda), L^{21}(\mu)\right\}=\frac{1}{\mu-\lambda}\left(L^{21}(\lambda)-L^{21}(\mu)\right)-L^{12}(\lambda),
\end{aligned}
$$

which is equivalent to the $r$-matrix algebra.

Proposition 2. $L(\lambda)$ satisfies the r-matrix relation

$$
\left\{L_{1}(\lambda), L_{2}(\mu)\right\}=\left[r_{12}(\lambda, \mu), L_{1}(\lambda)\right]-\left[r_{21}(\lambda, \mu), L_{2}(\mu)\right],
$$

where

$$
\begin{gathered}
r_{12}(\lambda, \mu)=r_{21}(\mu, \lambda)=\frac{1}{\mu-\lambda} P+S \\
P=\left(\begin{array}{llll}
1 & 0 & 0 & 0 \\
0 & 0 & 1 & 0 \\
0 & 1 & 0 & 0 \\
0 & 0 & 0 & 1
\end{array}\right), \quad S=\left(\begin{array}{llll}
0 & 0 & 0 & 0 \\
0 & 0 & 0 & 0 \\
0 & 0 & 0 & 0 \\
1 & 0 & 0 & 0
\end{array}\right) .
\end{gathered}
$$

Expand $\operatorname{det} L(\lambda)$ as follows:

$$
F_{\lambda} \triangleq \operatorname{det} L(\lambda)=\lambda+\sum_{m=0}^{\infty} F_{m} \lambda^{-m-1},
$$

where

$$
\begin{gathered}
F_{0}=-\frac{1}{2}\left\{\sum_{j=1}^{N} S_{j}^{+}-\sum_{j=1}^{N} \lambda_{j} S_{j}^{-}+\frac{1}{2}\left(\sum_{j=1}^{N} S_{j}^{-}\right)^{2}\right\}, \\
F_{m}=-\frac{1}{2}\left\{\sum_{j=1}^{N} \lambda_{j}^{m} S_{j}^{+}-\sum_{j=1}^{N} \lambda_{j}^{m+1} S_{j}^{-}\right. \\
\left.+\frac{1}{2}\left(\sum_{j=1}^{N} S_{j}^{-}\right)\left(\sum_{j=1}^{N} \lambda_{j}^{m} S_{j}^{-}\right)\right\} \\
-\frac{1}{4} \sum_{l+k=m-1}\left[\left(\sum_{j=1}^{N} \lambda_{j}^{l} S_{j}^{0}\right)\left(\sum_{j=1}^{N} \lambda_{j}^{k} S_{j}^{0}\right)\right. \\
\left.+\left(\sum_{j=1}^{N} \lambda_{j}^{l} S_{j}^{-}\right)\left(\sum_{j=1}^{N} \lambda_{j}^{k} S_{j}^{+}\right)\right], \quad m \geq 1 .
\end{gathered}
$$

Then, we have the involutive relation

$$
\left\{F_{j}, F_{k}\right\}=0, \quad j, k=0,1,2, \ldots
$$

Under the realization of (4), we obtain the following Lax matrix

$$
L(\lambda)=\left(\begin{array}{cc}
0 & 1 \\
-\lambda-\frac{1}{2}\langle q, q\rangle & 0
\end{array}\right)+\frac{1}{2} \sum_{j=1}^{N} \frac{1}{\lambda-\lambda_{j}}\left(\begin{array}{cc}
q_{j} p_{j} & -q_{j}^{2} \\
p_{j}^{2} & -q_{j} p_{j}
\end{array}\right) .
$$

Then, (21) becomes

$$
\begin{gathered}
F_{0}=-\frac{1}{2}\left\{\langle A q, q\rangle+\langle p, p\rangle+\frac{1}{2}\langle q, q\rangle^{2}\right\}, \\
F_{m}=-\frac{1}{2}\left\{\left\langle A^{m+1} q, q\right\rangle+\left\langle A^{m} p, p\right\rangle+\frac{1}{2}\langle q, q\rangle\left\langle A^{m} q, q\right\rangle\right\} \\
-\frac{1}{4} \sum_{l+k=m-1}\left[\left\langle A^{l} q, p\right\rangle\left\langle A^{k} q, p\right\rangle-\left\langle A^{l} q, q\right\rangle\left\langle A^{k} p, p\right\rangle\right],
\end{gathered}
$$$$
m \geq 1 \text {. }
$$

Here and after, $A=\operatorname{diag}\left(\lambda_{1}, \lambda_{2}, \ldots, \lambda_{N}\right)$ and $\langle$,$\rangle stands$ for the standard inner product in the Euclidean space. The Hamiltonian system with Hamiltonian $H=-F_{0}$ reads

$$
\begin{gathered}
q_{j, x}=p_{j}, \\
p_{j, x}=-\lambda_{j} q_{j}-\langle q, q\rangle q_{j}, \quad 1 \leq j \leq N,
\end{gathered}
$$

which is nothing but the Garnier system [27, 28]. We can check directly that the Garnier system (25) allows the Lax representation:

$$
\frac{d}{d x} L(\lambda)=[U(\lambda), L(\lambda)]
$$


where $L(\lambda)$ is given by (23), and

$$
U(\lambda)=\left(\begin{array}{cc}
0 & 1 \\
-\lambda-u & 0
\end{array}\right), \quad u=\langle q, q\rangle .
$$

Example 3 (The Garnier-Rosochatius System). From the realization of (7), we arrive at the Lax matrix

$$
\begin{aligned}
\widetilde{L}(\lambda)= & \left(\begin{array}{cc}
0 & 1 \\
-\lambda-\frac{1}{2}\langle q, q\rangle & 0
\end{array}\right) \\
& +\frac{1}{2} \sum_{j=1}^{N} \frac{1}{\lambda-\lambda_{j}}\left(\begin{array}{cc}
q_{j} p_{j} & -q_{j}^{2} \\
p_{j}^{2}+\gamma_{j} q_{j}^{-2} & -q_{j} p_{j}
\end{array}\right),
\end{aligned}
$$

and (21) becomes

$$
\begin{aligned}
\widetilde{F}_{0}=-\frac{1}{2}\left\{\langle A q, q\rangle+\langle p, p\rangle+\left\langle\gamma q^{-1}, q^{-1}\right\rangle+\frac{1}{2}\langle q, q\rangle^{2}\right\}, \\
\widetilde{F}_{m}=-\frac{1}{2}\left\{\left\langle A^{m+1} q, q\right\rangle+\left\langle A^{m} p, p\right\rangle+\left\langle A^{m} \gamma q^{-1}, q^{-1}\right\rangle\right. \\
+\frac{1}{2}\langle q, q\rangle\left\langle A^{m} q, q\right\rangle \\
+\frac{1}{2} \sum_{l+k=m-1}\left[\left\langle A^{l} q, p\right\rangle\left\langle A^{k} q, p\right\rangle-\left\langle A^{l} q, q\right\rangle\right. \\
\left.\left.\quad \times\left(\left\langle A^{k} p, p\right\rangle+\left\langle A^{k} \gamma q^{-1}, q^{-1}\right\rangle\right)\right]\right\}, \\
m \geq 1,
\end{aligned}
$$

where $\gamma=\operatorname{diag}\left(\gamma_{1}, \gamma_{2}, \ldots, \gamma_{N}\right)$. Choosing a Hamiltonian $\widetilde{H}=$ $-\widetilde{F}_{0}$, we arrive at

$$
\begin{array}{r}
q_{j, x}=p_{j}, \quad p_{j, x}=-\lambda_{j} q_{j}-\langle q, q\rangle q_{j}+\gamma_{j} q_{j}^{-3}, \\
1 \leq j \leq N,
\end{array}
$$

which is just the Garnier-Rosochatius system [8, 9, 13, 29]. It can be checked easily that (30) allows the Lax representation:

$$
\frac{d}{d x} \widetilde{L}(\lambda)=[U(\lambda), \widetilde{L}(\lambda)]
$$

where $\widetilde{L}(\lambda)$ is given by (28) and $U(\lambda)$ is given by (27).
Example 4 (The Second Rosochatius Deformation of the Garnier System). Based on the realization of (8), we obtain the Lax matrix

$$
\begin{aligned}
\widehat{L}(\lambda) & \\
= & \left(\begin{array}{cc}
0 & 1 \\
-\lambda-\frac{1}{2}\left(\langle q, q\rangle+\sum_{i=1}^{N} \beta_{i}\left(p_{i}^{2}+\gamma_{i} q_{i}^{-2}\right)^{-1}\right) & 0
\end{array}\right) \\
& +\frac{1}{2} \sum_{j=1}^{N} \frac{1}{\lambda-\lambda_{j}} \\
& \times\left(\begin{array}{cc}
q_{j} p_{j} & -q_{j}^{2}-\beta_{j}\left(p_{j}^{2}+\gamma_{j} q_{j}^{-2}\right)^{-1} \\
p_{j}^{2}+\gamma_{j} q_{j}^{-2} & -q_{j} p_{j}
\end{array}\right),
\end{aligned}
$$

and the integrals of motion

$$
\begin{aligned}
& \widehat{F}_{0}=-\frac{1}{2}\left\{\langle A q, q\rangle+\sum_{j=1}^{N} \lambda_{j} \beta_{j}\left(p_{j}^{2}+\gamma_{j} q_{j}^{-2}\right)^{-1}\right. \\
& +\langle p, p\rangle+\left\langle\gamma q^{-1}, q^{-1}\right\rangle \\
& \left.+\frac{1}{2}\left(\langle q, q\rangle+\sum_{j=1}^{N} \beta_{j}\left(p_{j}^{2}+\gamma_{j} q_{j}^{-2}\right)^{-1}\right)^{2}\right\}, \\
& \widehat{F}_{m}=-\frac{1}{2}\left\{\left\langle A^{m+1} q, q\right\rangle\right. \\
& +\sum_{j=1}^{N} \lambda_{j}^{m+1} \beta_{j}\left(p_{j}^{2}+\gamma_{j} q_{j}^{-2}\right)^{-1} \\
& +\left\langle A^{m} p, p\right\rangle+\left\langle A^{m} \gamma q^{-1}, q^{-1}\right\rangle \\
& +\frac{1}{2}\left(\langle q, q\rangle+\sum_{j=1}^{N} \beta_{j}\left(p_{j}^{2}+\gamma_{j} q_{j}^{-2}\right)^{-1}\right) \\
& \times\left(\left\langle A^{m} q, q\right\rangle+\sum_{j=1}^{N} \lambda_{j}^{m} \beta_{j}\left(p_{j}^{2}+\gamma_{j} q_{j}^{-2}\right)^{-1}\right) \\
& +\frac{1}{2} \sum_{l+k=m-1}\left[\left\langle A^{l} q, p\right\rangle\left\langle A^{k} q, p\right\rangle\right.
\end{aligned}
$$

$$
\begin{aligned}
& -\left(\left\langle A^{l} q, q\right\rangle\right. \\
& \left.+\sum_{j=1}^{N} \lambda_{j}^{l} \beta_{j}\left(p_{j}^{2}+\gamma_{j} q_{j}^{-2}\right)^{-1}\right) \\
& \left.\left.\times\left(\left\langle A^{k} p, p\right\rangle+\left\langle A^{k} \gamma q^{-1}, q^{-1}\right\rangle\right)\right]\right\} .
\end{aligned}
$$


Choosing a Hamiltonian $\widehat{H}=-\widehat{F}_{0}$, we obtain an integrable Hamiltonian system

$$
\begin{aligned}
q_{j, x}= & p_{j}-\left(\lambda_{j}+\langle q, q\rangle+\sum_{i=1}^{N} \beta_{i}\left(p_{i}^{2}+\gamma_{i} q_{i}^{-2}\right)^{-1}\right) \\
& \times \beta_{j}\left(p_{j}^{2}+\gamma_{j} q_{j}^{-2}\right)^{-2} p_{j}, \\
p_{j, x}= & \gamma_{j} q_{j}^{-3}-\left(\lambda_{j}+\langle q, q\rangle+\sum_{i=1}^{N} \beta_{i}\left(p_{i}^{2}+\gamma_{i} q_{i}^{-2}\right)^{-1}\right) \\
& \times\left(q_{j}+\beta_{j} \gamma_{j}\left(p_{j}^{2}+\gamma_{j} q_{j}^{-2}\right)^{-2} q_{j}^{-3}\right),
\end{aligned}
$$

which is the second Rosochatius deformation of the Garnier system. With direct calculations, we find that (35) admits the Lax representation:

$$
\frac{d}{d x} \widehat{L}(\lambda)=[\widehat{U}(\lambda), \widehat{L}(\lambda)]
$$

where

$$
\begin{aligned}
\widehat{U}(\lambda) & =\left(\begin{array}{cr}
0 & 1 \\
-\lambda-\widehat{u} & 0
\end{array}\right), \\
\widehat{u} & =\langle q, q\rangle+\sum_{j=1}^{N} \beta_{j}\left(p_{j}^{2}+\gamma_{j} q_{j}^{-2}\right)^{-1} .
\end{aligned}
$$

There is no doubt that we can consecutively construct Rosochatius deformations of the Garnier system by applying the two kinds of realizations in Proposition 1 in turn and the recipe we described in Section 2.2. Here, we only present the above two examples.

\section{Consecutive Rosochatius Deformations of the Hénon-Heiles System}

Now, we begin with the Lax matrix of the form

$$
\begin{aligned}
L(\lambda)= & \left(\begin{array}{cc}
2 p_{N+1} & 8 \lambda-4 q_{N+1} \\
-8 \lambda^{2}-4 q_{N+1} \lambda-2 q_{N+1}^{2}+\frac{1}{2} \sum_{j=1}^{N} S_{j}^{-} & -2 p_{N+1}
\end{array}\right) \\
& +\frac{1}{2} \sum_{j=1}^{N} \frac{1}{\lambda-\lambda_{j}}\left(\begin{array}{cc}
S_{j}^{0} & S_{j}^{-} \\
S_{j}^{+} & -S_{j}^{0}
\end{array}\right) .
\end{aligned}
$$

Defining a generating function

$$
F_{\lambda}=\operatorname{det} L(\lambda)=64 \lambda^{3}+P_{0}+\sum_{m=0}^{\infty} F_{m} \lambda^{-m-1}
$$

we have

$$
\begin{aligned}
P_{0}= & -4 p_{N+1}^{2}-8 q_{N+1}^{3}-4 \sum_{j=1}^{N} S_{j}^{+}+4 q_{N+1} \sum_{j=1}^{N} S_{j}^{-}+4 \sum_{j=1}^{N} \lambda_{j} S_{j}^{-}, \\
F_{0}= & -4 \sum_{j=1}^{N} \lambda_{j} S_{j}^{+}+4 \sum_{j=1}^{N} \lambda_{j}^{2} S_{j}^{-}+2 q_{N+1}\left(\sum_{j=1}^{N} S_{j}^{+}+\sum_{j=1}^{N} \lambda_{j} S_{j}^{-}\right) \\
& +q_{N+1}^{2} \sum_{j=1}^{N} S_{j}^{-}-2 p_{N+1} \sum_{j=1}^{N} S_{j}^{0}-\frac{1}{4}\left(\sum_{j=1}^{N} S_{j}^{-}\right)^{2} \\
F_{m}= & -4 \sum_{j=1}^{N} \lambda_{j}^{m+1} S_{j}^{+} \\
& +4 \sum_{j=1}^{N} \lambda_{j}^{m+2} S_{j}^{-}+2 q_{N+1}\left(\sum_{j=1}^{N} \lambda_{j}^{m} S_{j}^{+}+\sum_{j=1}^{N} \lambda_{j}^{m+1} S_{j}^{-}\right) \\
& +q_{N+1}^{2} \sum_{j=1}^{N} \lambda_{j}^{m} S_{j}^{-} \\
& -\frac{1}{4} \sum_{l+k=m-1}\left[\left(\sum_{j=1}^{N} \lambda_{j}^{l} S_{j}^{0}\right)\left(\sum_{j=1}^{N} \lambda_{j}^{k} S_{j}^{0}\right)^{N} \sum_{j=1}^{N} \lambda_{j}^{l} S_{j}^{-}\right)\left(\sum_{j=1}^{N} \lambda_{j}^{k} S_{j}^{+}\right) \\
& -2 p_{N+1} \sum_{j=1}^{m} \lambda_{j}^{m} S_{j}^{0}-\frac{1}{4}\left(\sum_{j=1}^{N} S_{j}^{-}\right)\left(\sum_{j=1}^{N} \lambda_{j}^{m} S_{j}^{-}\right)
\end{aligned}
$$

We may check directly that (38) satisfies the same $r$-matrix relation as (18). Thus, we have the involutive relation:

$$
\left\{P_{0}, F_{k}\right\}=\left\{F_{j}, F_{k}\right\}=0, \quad j, k=0,1,2, \ldots
$$

Now, we discuss the integrable Hamiltonian system generated by the Lax matrix (38) and its realizations. Firstly, with the realization of (4), we arrive at the following Lax matrix:

$$
\begin{aligned}
L(\lambda)= & \left(\begin{array}{cc}
2 p_{N+1} & 8 \lambda-4 q_{N+1} \\
-8 \lambda^{2}-4 q_{N+1} \lambda-2 q_{N+1}^{2}-\frac{1}{2}\langle q, q\rangle & -2 p_{N+1}
\end{array}\right) \\
& +\frac{1}{2} \sum_{j=1}^{N} \frac{1}{\lambda-\lambda_{j}}\left(\begin{array}{cc}
q_{j} p_{j} & -q_{j}^{2} \\
p_{j}^{2} & -q_{j} p_{j}
\end{array}\right),
\end{aligned}
$$


and (40) becomes

$$
\begin{aligned}
P_{0}= & -4 p_{N+1}^{2}-8 q_{N+1}^{3}-4\langle p, p\rangle-4 q_{N+1}\langle q, q\rangle-4\langle q, A q\rangle, \\
F_{0}= & -4\langle p, A p\rangle-4\left\langle q, A^{2} q\right\rangle+2 q_{N+1}(\langle p, p\rangle-\langle q, A q\rangle) \\
& -q_{N+1}^{2}\langle q, q\rangle-2 p_{N+1}\langle q, p\rangle-\frac{1}{4}\langle q, q\rangle^{2}, \\
F_{m}= & -4\left\langle p, A^{m+1} p\right\rangle-4\left\langle q, A^{m+2} q\right\rangle \\
& +2 q_{N+1}\left(\left\langle p, A^{m} p\right\rangle-\left\langle q, A^{m+1} q\right\rangle\right) \\
& -q_{N+1}^{2}\left\langle q, A^{m} q\right\rangle-2 p_{N+1}\left\langle q, A^{m} p\right\rangle-\frac{1}{4}\langle q, q\rangle\left\langle q, A^{m} q\right\rangle \\
& -\frac{1}{4} \sum_{l+k=m-1}\left[\left\langle A^{l} q, p\right\rangle\left\langle A^{k} q, p\right\rangle-\left\langle A^{l} q, q\right\rangle\left\langle A^{k} p, p\right\rangle\right], \\
& m \geq 1 .
\end{aligned}
$$
reads

The Hamiltonian system with Hamiltonian $H=-(1 / 8) P_{0}$

$$
\begin{gathered}
q_{j, x}=p_{j}, \\
p_{j, x}=-\lambda_{j} q_{j}-q_{N+1} q_{j}, \\
q_{N+1, x}=p_{N+1}, \\
p_{N+1, x}=-3 q_{N+1}^{2}-\frac{1}{2}\langle q, q\rangle, \quad 1 \leq j \leq N,
\end{gathered}
$$

which is just the Hénon-Heiles system [30-32], and it allows the Lax representation:

$$
\frac{d}{d x} L(\lambda)=[U(\lambda), L(\lambda)]
$$

where $L(\lambda)$ is given by (42), and

$$
U(\lambda)=\left(\begin{array}{cc}
0 & 1 \\
-\lambda-q_{N+1} & 0
\end{array}\right)
$$

Example 5 (The Rosochatius Deformation of the HénonHeiles System). Under realization of (7), we arrive at the Lax matrix

$$
\begin{aligned}
\tilde{L}(\lambda)= & \left(\begin{array}{cc}
2 p_{N+1} & 8 \lambda-4 q_{N+1} \\
-8 \lambda^{2}-4 q_{N+1} \lambda-2 q_{N+1}^{2}-\frac{1}{2}\langle q, q\rangle & -2 p_{N+1}
\end{array}\right) \\
& +\frac{1}{2} \sum_{j=1}^{N} \frac{1}{\lambda-\lambda_{j}}\left(\begin{array}{cc}
q_{j} p_{j} & -q_{j}^{2} \\
p_{j}^{2}+\gamma_{j} q_{j}^{-2} & -q_{j} p_{j}
\end{array}\right),
\end{aligned}
$$

and (40) becomes

$$
\begin{aligned}
\widetilde{P}_{0}= & -4 p_{N+1}^{2}-8 q_{N+1}^{3}-4\left(\langle p, p\rangle+\left\langle q^{-1}, \gamma q^{-1}\right\rangle\right) \\
& -4 q_{N+1}\langle q, q\rangle-4\langle q, A q\rangle \\
\widetilde{F}_{0}= & -4\left(\langle p, A p\rangle+\left\langle q^{-1}, A \gamma q^{-1}\right\rangle\right)-4\left\langle q, A^{2} q\right\rangle \\
& +2 q_{N+1}\left(\langle p, p\rangle+\left\langle q^{-1}, \gamma q^{-1}\right\rangle-\langle q, A q\rangle\right) \\
& -q_{N+1}^{2}\langle q, q\rangle-2 p_{N+1}\langle q, p\rangle-\frac{1}{4}\langle q, q\rangle^{2}, \\
\widetilde{F}_{m}= & -4\left(\left\langle p, A^{m+1} p\right\rangle+\left\langle q^{-1}, A^{m+1} \gamma q^{-1}\right\rangle\right)-4\left\langle q, A^{m+2} q\right\rangle \\
& -2 p_{N+1}\left\langle q, A^{m} p\right\rangle-\frac{1}{4}\langle q, q\rangle\left\langle q, A^{m} q\right\rangle \\
& +2 q_{N+1}\left(\left\langle p, A^{m} p\right\rangle+\left\langle q^{-1}, A^{m} \gamma q^{-1}\right\rangle-\left\langle q, A^{m+1} q\right\rangle\right) \\
& -q_{N+1}^{2}\left\langle q, A^{m} q\right\rangle \\
& -\frac{1}{4} \sum_{l+k=m-1}\left[\left\langle A^{l} q, p\right\rangle\left\langle A^{k} q, p\right\rangle\right. \\
& \left.-\left\langle A^{l} q, q\right\rangle\left(\left\langle A^{k} p, p\right\rangle+\left\langle A^{k} \gamma q^{-1}, q^{-1}\right\rangle\right)\right]
\end{aligned}
$$

Taking the Hamiltonian as $\widetilde{H}=-(1 / 8) \widetilde{P}_{0}$, we have

$$
\begin{gathered}
q_{j, x}=p_{j}, \\
p_{j, x}=-\lambda_{j} q_{j}-q_{N+1} q_{j}+\gamma_{j} q_{j}^{-3}, \\
q_{N+1, x}=p_{N+1}, \\
p_{N+1, x}=-3 q_{N+1}^{2}-\frac{1}{2}\langle q, q\rangle, \quad 1 \leq j \leq N,
\end{gathered}
$$

which is exactly the Rosochatius deformation of HénonHeiles system [12, 15]. It can be checked directly that (49) allows the Lax representation:

$$
\frac{d}{d x} \widetilde{L}(\lambda)=[U(\lambda), \widetilde{L}(\lambda)]
$$

where $\widetilde{L}(\lambda)$ is given by (47) and $U(\lambda)$ is given by (46).

Example 6 (The Second Rosochatius Deformation of the Hénon-Heiles System). Based on the realization of (8), we obtain the Lax matrix 


$$
\begin{aligned}
& \widehat{L}(\lambda)=\left(\begin{array}{cc}
2 p_{N+1} & 8 \lambda-4 q_{N+1} \\
-8 \lambda^{2}-4 q_{N+1} \lambda-2 q_{N+1}^{2}-\frac{1}{2}\left(\langle q, q\rangle+\sum_{j=1}^{N} \beta_{j}\left(p_{j}^{2}+\gamma_{j} q_{j}^{-2}\right)^{-1}\right)^{-2 p_{N+1}}
\end{array}\right) \\
& +\frac{1}{2} \sum_{j=1}^{N} \frac{1}{\lambda-\lambda_{j}}\left(\begin{array}{cc}
q_{j} p_{j} & -q_{j}^{2}-\beta_{j}\left(p_{j}^{2}+\gamma_{j} q_{j}^{-2}\right)^{-1} \\
p_{j}^{2}+\gamma_{j} q_{j}^{-2} & -q_{j} p_{j}
\end{array}\right) .
\end{aligned}
$$

The integrals of motion $\widehat{P}_{0}, \widehat{F}_{0}$, and $\widehat{F}_{m}, m \geq 1$, can be generated from $\operatorname{det} \widehat{L}(\lambda)$. In particular, we have

$$
\begin{aligned}
\widehat{P}_{0}= & -4 p_{N+1}^{2}-8 q_{N+1}^{3}-4\left(\langle p, p\rangle+\left\langle q^{-1}, \gamma q^{-1}\right\rangle\right) \\
& -4 q_{N+1}\left(\langle q, q\rangle+\sum_{j=1}^{N} \beta_{j}\left(p_{j}^{2}+\gamma_{j} q_{j}^{-2}\right)^{-1}\right) \\
& -4\left(\langle q, A q\rangle+\sum_{j=1}^{N} \lambda_{j} \beta_{j}\left(p_{j}^{2}+\gamma_{j} q_{j}^{-2}\right)^{-1}\right) .
\end{aligned}
$$

Choosing a Hamiltonian as $\widehat{H}=-(1 / 8) \widehat{P}_{0}$, we arrive at an integrable Hamiltonian system

$$
\begin{gathered}
q_{j, x}=p_{j}-\left(\lambda_{j}+q_{N+1}\right) \beta_{j}\left(p_{j}^{2}+\gamma_{j} q_{j}^{-2}\right)^{-2} p_{j}, \\
p_{j, x}=-\lambda_{j} q_{j}-q_{N+1} q_{j}+\gamma_{j} q_{j}^{-3} \\
-\left(\lambda_{j}+q_{N+1}\right) \beta_{j} \gamma_{j}\left(p_{j}^{2}+\gamma_{j} q_{j}^{-2}\right)^{-2} q_{j}^{-3}, \\
q_{N+1, x}=p_{N+1}, \\
p_{N+1, x}=-3 q_{N+1}^{2}-\frac{1}{2}\left(\langle q, q\rangle+\sum_{i=1}^{N} \beta_{i}\left(p_{i}^{2}+\gamma_{i} q_{i}^{-2}\right)^{-1}\right), \\
1 \leq j \leq N,
\end{gathered}
$$

which is the second Rosochatius deformation of HénonHeiles system. Again, we may check that (53) allows the Lax representation:

$$
\frac{d}{d x} \widehat{L}(\lambda)=[U(\lambda), \widehat{L}(\lambda)]
$$

where $\widehat{L}(\lambda)$ is given by $(51)$ and $U(\lambda)$ is given by (46).

\section{Concluding Remarks}

We have shown how to consecutively generate integrable Rosochatius deformations of the integrable Hamiltonian systems whose Lax matrices are of the form of the generalized Gaudin magnet. As applications, we obtained the consecutive Rosochatius deformations of the Garnier system and the Hénon-Heiles system together with their Lax representations. Our method is performed in a unified way. There is no doubt that our method can be applied to other constrained soliton flows [28, 32] whose Lax matrices are of the form of the generalized Gaudin magnet or the generalized Gaudin magnet with boundary. Also, we remark that our method can be used to construct consecutive Rosochatius deformations of the integrable symplectic maps and the soliton equations with self-consistent sources.

\section{Conflict of Interests}

The authors declare that there is no conflict of interests regarding the publication of this paper.

\section{Acknowledgments}

This work was partially supported by the National Natural Science Foundation of China (Grant nos. 11301229 and 11271168), the Natural Science Foundation of the Jiangsu Province (Grant no. BK20130224), and the Natural Science Foundation of the Jiangsu Higher Education Institutions of China (Grant no. 13KJB110009).

\section{References}

[1] E. Rosochatius, " Uber die Bewegung eines Punktes," [Ph.D. dissertation], University of Gotingen, 1877.

[2] C. Neumann, "De problemate quodam mechanico, quod ad primam integralium ultraellipticorum classem revocatur," Journal für die Reine und Angewandte Mathematik, vol. 56, pp. 46-63, 1859.

[3] J. Moser, "Geometry of quadrics and spectral theory," in The Chern Symposium 1979, pp. 147-188, Springer, New York, NY, USA, 1980.

[4] M. R. Adams, J. Harnad, and E. Previato, "Isospectral hamiltonian flows in finite and infinite dimensions. I: generalized Moser systems and moment maps into loop algebras," Communications in Mathematical Physics, vol. 117, no. 3, pp. 451-500, 1988.

[5] J. Harnad and P. Winternitz, "Classical and quantum integrable systems in 263-1263-1263-1 and separation of variables," Communications in Mathematical Physics, vol. 172, no. 2, pp. 263285, 1995.

[6] C. Bartocci, G. Falqui, and M. Pedroni, "A geometric approach to the separability of the Neumann-Rosochatius system," Differential Geometry and Its Application, vol. 21, no. 3, pp. 349-360, 2004 . 
[7] C.-W. Cao and B.-Q. Xia, "From Rosochatius system to KdV equation," Communications in Theoretical Physics, vol. 54, no. 4, pp. 619-624, 2010.

[8] S. Wojciechowski, "Integrability of one particle in a perturbed central quartic potential," Physica Scripta, vol. 31, no. 6, pp. 433$438,1985$.

[9] G. Tondo, "On the integrability of stationary and restricted flows of the KdV hierarchy," Journal of Physics A, vol. 28, no. 17, pp. 5097-5115, 1995.

[10] R. Kubo, W. Ogura, T. Saito, and Y. Yasui, “The Gauss-Knörrer map for the Rosochatius dynamical system," Physics Letters A, vol. 251, no. 1, pp. 6-12, 1999.

[11] Y. B. Suris, "Discrete-time analogues of some nonlinear oscillators in the inverse-square potential," Journal of Physics A, vol. 27, no. 24, pp. 8161-8169, 1994.

[12] A. N. W. Hone, V. B. Kuznetsov, and O. Ragnisco, "Bäcklund transformations for many-body systems related to KdV," Journal of Physics A, vol. 32, no. 27, pp. L299-L306, 1999.

[13] G. Zhou, "Perturbation expansion and Nth order Fermi golden rule of the nonlinear Schrödinger equations," Journal of Mathematical Physics, vol. 48, no. 5, Article ID 053509, 23 pages, 2007.

[14] B. Xia and R. Zhou, "Integrable deformations of integrable symplectic maps," Physics Letters A, vol. 373, no. 47, pp. 43604367, 2009.

[15] Y. Q. Yao and Y. B. Zeng, "Integrable rosochatius deformations of higher-order constrained flows and the soliton hierarchy with self-consistent sources," Journal of Physics A, vol. 41, no. 29, Article ID 295205, 2008.

[16] H. Dimov and R. C. Rashkov, "A note on spin chain/string duality," International Journal of Modern Physics A, vol. 20, no. 18, pp. 4337-4353, 2005.

[17] P. Bozhilov, "Neumann and Neumann-Rosochatius integrable systems from membranes on $\mathrm{Ad}_{4} \times \mathrm{S}^{7}$," Journal of High Energy Physics, no. 8, article 073, 21 pages, 2007.

[18] N. A. Kostov, "Quasi-periodic solutions of the integrable dynamical systems related to Hill's equation," Letters in Mathematical Physics A, vol. 17, no. 2, pp. 95-108, 1989.

[19] P. L. Christiansen, J. C. Eilbeck, V. Z. Enolskii, and N. A. Kostov, "Quasi-periodic and periodic solutions for coupled nonlinear Schrödinger equations of Manakov type," Proceedings of the Royal Society of London A, vol. 456, no. 2001, pp. 2263-2281, 2000.

[20] Y. Yao and Y. Zeng, "The Bi-hamiltonian structure and new solutions of KdV6 equation," Letters in Mathematical Physics, vol. 86, no. 2-3, pp. 193-208, 2008.

[21] B. Q. Xia and R. G. Zhou, "Consecutive Rosochatius deformations of the Neumann system," Journal of Mathematical Physics, vol. 54, no. 10, Article ID 103514, 2013.

[22] V. B. Kuznetsov, "Quadrics on real Riemannian spaces of constant curvature: separation of variables and connection with Gaudin magnet," Journal of Mathematical Physics, vol. 33, no. 9, pp. 3240-3254, 1992.

[23] J. C. Eilbeck, V. Z. Ėnolskiǔ, V. B. Kuznetsov, and A. V. Tsiganov, "Linear $r$-matrix algebra for classical separable systems," Journal of Physics A, vol. 27, no. 2, pp. 567-578, 1994.

[24] O. Babelon and C.-M. Viallet, "Hamiltonian structures and Lax equations," Physics Letters B, vol. 237, no. 3-4, pp. 411-416, 1990.

[25] Z. Qiao, "Generalized $r$-matrix structure and algebro-geometric solution for integrable system," Reviews in Mathematical Physics $A$, vol. 13 , no. 5 , pp. 545-586, 2001.
[26] V. I. Arnold, Mathematical Methods of Classical Mechanics, Springer, Berlin, Germany, 1978.

[27] R. Garnier, "Sur une classe de systèmes différentiels abéliens déduits de la théorie des équations linéaires," Rendiconti del Circolo Matematico di Palermo, vol. 43, no. 1, pp. 155-191, 1919.

[28] C. W. Cao and X. G. Geng, "Classical integrable systems generated through nonlinearization of eigenvalue problems," in Nonlinear Physics, C. H. Gu, Y. S. Li, and G. Z. Tu, Eds., Research Reports in Physics, pp. 66-78, Springer, Berlin, Germany, 1990.

[29] B. Xia, "A hierarchy of Garnier-Rosochatius systems," Journal of Mathematical Physics, vol. 52, no. 6, Article ID 063506, 11 pages, 2011.

[30] M. Hénon and C. Heiles, "The applicability of the third integral of motion: some numerical experiments," Astronomical Journal, vol. 69, p. 73, 1964.

[31] Y. F. Chang, M. Tabor, and J. Weiss, "Analytic structure of the Hénon-Heiles Hamiltonian in integrable and nonintegrable regimes," Journal of Mathematical Physics, vol. 23, no. 4, pp. 531538, 1982.

[32] M. Antonowicz and S. Rauch-Wojciechowski, "Bi-Hamiltonian formulation of the Hénon-Heiles system and its multidimensional extensions," Physics Letters A, vol. 163, no. 3, pp. 167-172, 1992. 


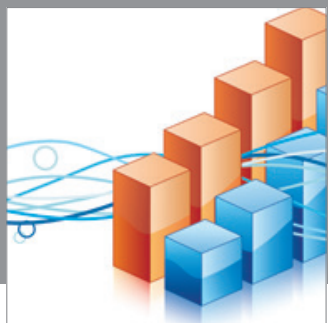

Advances in

Operations Research

mansans

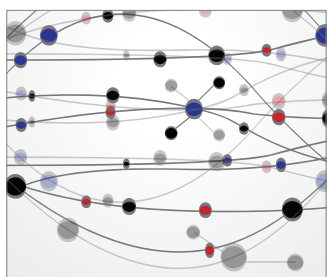

The Scientific World Journal
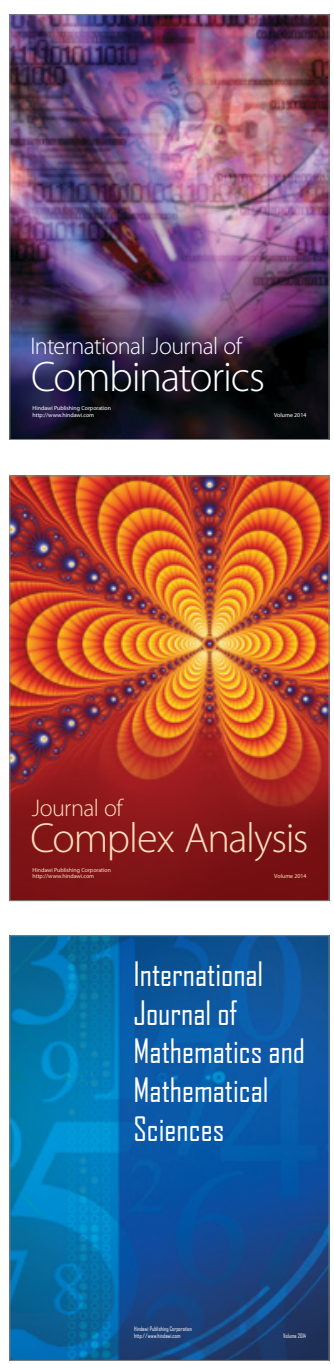
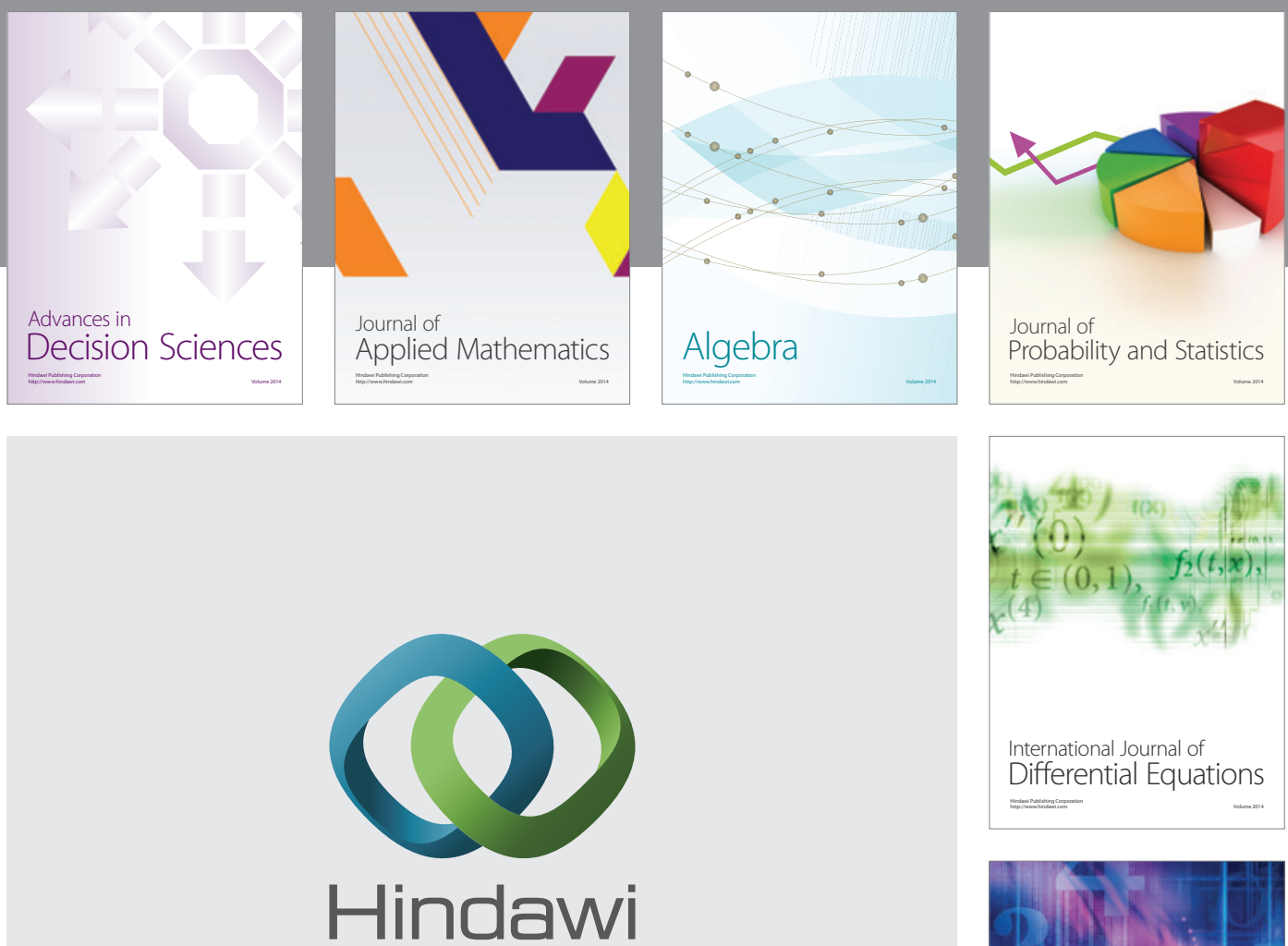

Submit your manuscripts at http://www.hindawi.com
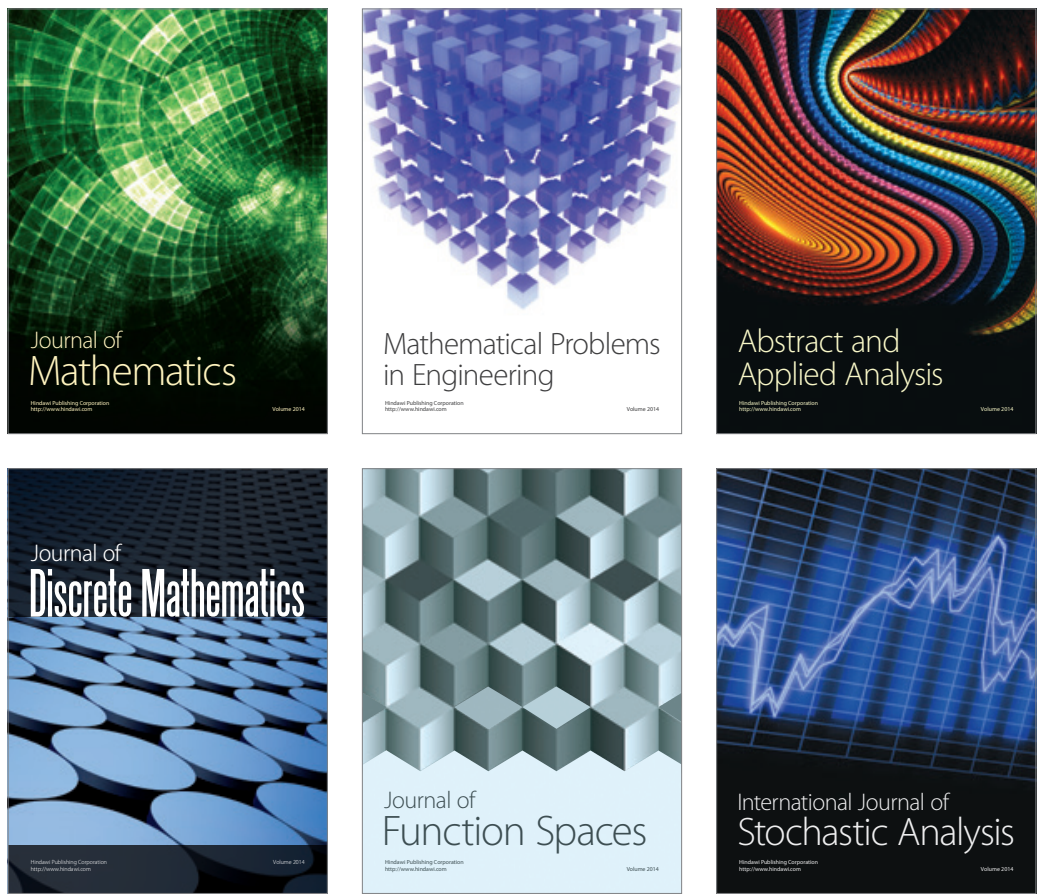

Journal of

Function Spaces

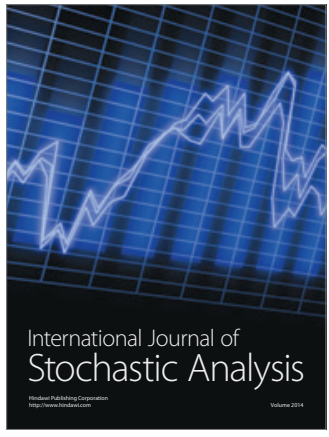

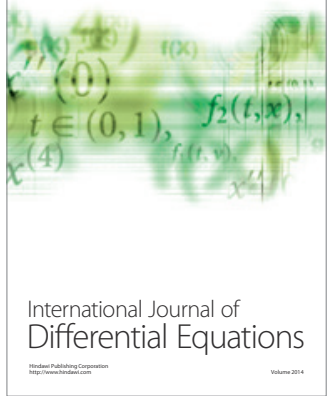
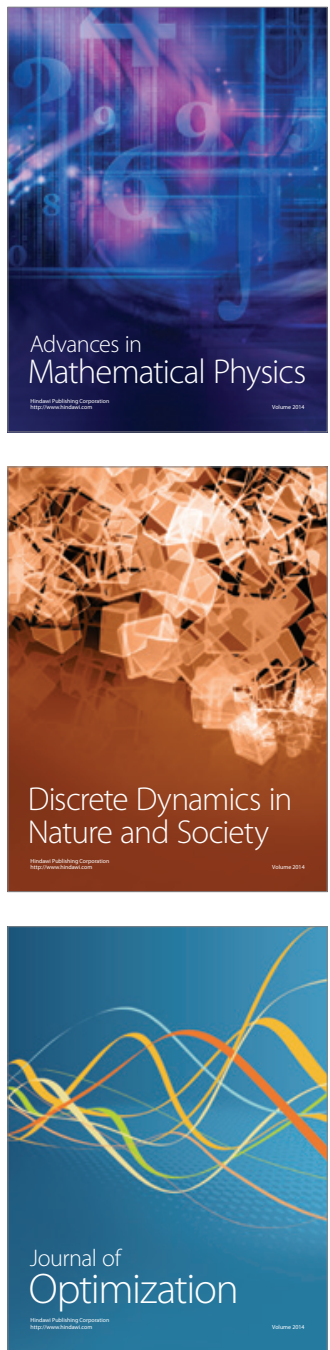